\title{
Joint effects of uric acid and lymphocyte count on adverse outcomes in elderly patients with rheumatic heart disease undergoing valve replacement surgery
}

Xue-biao Wei, MD, ${ }^{\mathrm{a}, \mathrm{b}}$ Wei-jian Chen, MD,${ }^{\mathrm{c}}$ Chong-yang Duan, MD, PhD, ${ }^{\mathrm{d}}$ Tie-he Qin, MD, ${ }^{\mathrm{a}}$ Yang Yu, MD, ${ }^{\mathrm{e}}$ Qing-shan Geng, $\mathrm{MD}, \mathrm{PhD},{ }^{\mathrm{a}, \mathrm{b}}$ and Lei Jiang, $\mathrm{MD}, \mathrm{PhD}^{\mathrm{a}, \mathrm{b}}$

\section{ABSTRACT}

Objectives: Increased uric acid and decreased lymphocyte count are common in elderly patients or those with heart failure, which were prognostic markers. We aimed to investigate the joint effect of uric acid and lymphocyte count for risk stratification in elderly patients with rheumatic heart disease undergoing valve replacement surgery.

Methods: Uric acid to lymphocyte ratio was calculated as serum uric acid $(\mathrm{mg} / \mathrm{dL}) / \mathrm{lym}$ hocyte count $\left(\times 10^{9} / \mathrm{L}\right)$. Univariate and multivariate analyses were performed to investigate the association of uric acid to lymphocyte ratio, with adverse events in 949 elderly patients with rheumatic heart disease undergoing valve replacement surgery. For clinical use, the uric acid to lymphocyte ratio was classified into 3 groups by the tertile, and a cutoff was also selected according to the receiver operator characteristic curve.

Results: Uric acid to lymphocyte ratio produced relatively higher predictive value (area under the curve, 0.703; 95\% confidence interval [CI], 0.630-0.776; $P<.001)$ than uric acid or lymphocyte count for in-hospital mortality, and the optimal cutoff was 3.7 (sensitivity, $82.1 \%$; specificity, 52.4\%). Uric acid to lymphocyte ratio was an independent predictor for in-hospital (adjusted odds ratio, $1.17 ; 95 \%$ CI, 1.07-1.29; $P=.001$ ) and 1-year mortality (adjusted hazard ratio, $1.13 ; 95 \% \mathrm{CI}, 1.03-1.25 ; P=.010)$. The in-hospital mortality increased from the lowest to the highest uric acid to lymphocyte ratio tertile $(P<.001)$ and significantly higher in patients with uric acid to lymphocyte ratio greater than 3.7 $(P<.001)$. The cumulative 1 -year postoperative mortality risk was significantly higher in patients with uric acid to lymphocyte ratio greater than $3.7(P<.001)$ or upper uric acid to lymphocyte ratio tertile $(P<.001)$.

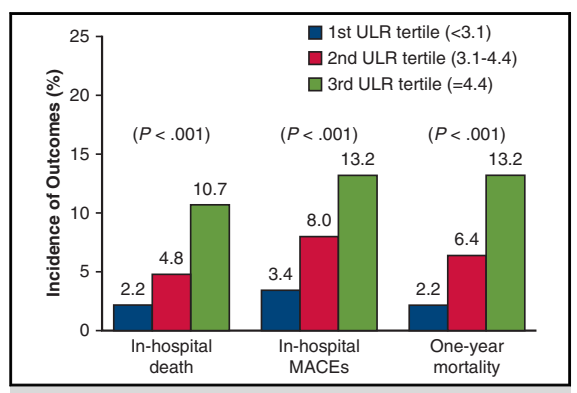

Association of ULR with adverse outcomes.

\section{Central Message}

ULR, as a novel index, was independently associated with in-hospital and 1-year mortality in elderly patients with RHD undergoing VRS

\section{Perspective}

ULR combining 2 distinct phenomena-hyperuricemia and reduced lymphocyte countcould be considered as a relatively powerful preoperative risk assessment tool in elderly patients with RHD undergoing VRS. Emphasis should be placed on those patients with preVRS high ULR.

See Commentary on page 428 .

Conclusions: Uric acid to lymphocyte ratio, combining the effect of uric acid and lymphocyte count, produced more prognostic value in elderly patients with rheumatic heart disease undergoing valve replacement surgery, which could be considered as a preoperative risk-stratified method. (J Thorac Cardiovasc Surg 2019;158:420-7)

From the ${ }^{\mathrm{a}}$ Guangdong Geriatrics Institute, Guangdong General Hospital, Guangdong Academy of Medical Sciences, and ${ }^{\mathrm{b}}$ Department of Cardiology, Guangdong Cardiovascular Institute, Guangdong General Hospital, Guangdong Academy of Medical Sciences, Guangzhou, China; ${ }^{\mathrm{c}}$ Department of Cardiology, Shunde Hospital of Guangzhou Medical University, Foshan, China; ${ }^{\mathrm{d} D e p a r t m e n t ~ o f ~ B i o s t a t i s t i c s, ~}$ School of Public Health, Southern Medical University, Guangzhou, China; and ${ }^{\mathrm{e}}$ Department of Biomedical Engineering, School of Medicine, University of Alabama at Birmingham, Birmingham, Ala.

This study was supported by Science and Technology Projects of Guangzhou (grant no. 201704020124) and Science and Technology Projects of Guangdong (grant no. 2016A030313791). The funders had no role in the study design, data collection and analysis, decision to publish, or preparation of the manuscript. The work was not funded by any industry sponsors.

X-b.W., W-j.C., and C-y.D. are considered as co-first authors.

Received for publication May 12, 2018; revisions received Aug 14, 2018; accepted for publication Oct 9, 2018; available ahead of print Nov 17, 2018.

Address for reprints: Lei Jiang, MD, PhD, and Tie-he Qin, MD, Department of Geriatrics, Guangdong Geriatrics Institute, Guangdong General Hospital, Guangdong Academic of Medical Sciences, 106 Zhongshan Road 2, Guangzhou 510080, Guangdong, China (E-mail: jianglei@smu.edu.cn and qintiehe@163.com). $0022-5223 / \$ 36.00$

Copyright (C) 2018 by The American Association for Thoracic Surgery https://doi.org/10.1016/j.jtcvs.2018.10.058 


\section{Abbreviations and Acronyms \\ AUC = area under the curve \\ $\mathrm{CI}=$ confidence interval \\ eGFR = estimated glomerular filtration rate \\ euroSCORE $=$ European System for Cardiac \\ Operative Risk Evaluation

$\begin{array}{ll}\text { HR } & =\text { hazard ratio } \\ \text { LVEF } & =\text { left ventricular ejection fraction } \\ \text { MACE } & =\text { major adverse clinical events } \\ \text { NYHA } & =\text { New York Heart Association } \\ \text { OR } & =\text { odds ratio } \\ \text { PSP } & =\text { pulmonary systolic pressure } \\ \text { RHD } & =\text { rheumatic heart disease } \\ \text { ROC } & =\text { receiver operating characteristic } \\ \text { RV } & =\text { right ventricular } \\ \text { ULR } & =\text { uric acid to lymphocyte count ratio } \\ \text { VRS } & =\text { valve replacement surgery }\end{array}$

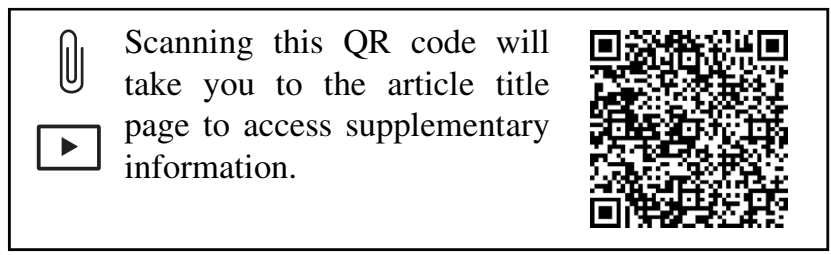

Rheumatic heart disease (RHD) is a chronic acquired heart disease caused by an autoimmune response to group A streptococcal infection, which can lead to valve damage, heart failure, and death. ${ }^{1}$ This naturally creates an enormous social and economic burden in developing countries. Valve replacement surgery (VRS) is recommended when symptoms appear in patients with severe valve damage. ${ }^{2}$ However, the early and late postoperative mortality remain high, especially among elderly patients, ${ }^{3,4}$ and few riskstratified tools were emphasized in this aged population. Therefore, preoperative evaluation is still necessary to identify those patients with a high mortality risk and accordingly make safe and effective clinical decisions to improve prognosis.

Increased uric acid is common in patients with reduced or preserved ejection fraction heart failure, which is due to inflammation, oxidative stress, and cardiorenal syndrome. $^{5-7}$ Increasing research has shown that hyperuricemia is a predictor for poor outcomes in heart failure. ${ }^{8-10}$ On the other hand, activated neuroendocrine and inflammatory systems in heart failure might decrease the lymphocyte count. ${ }^{11}$ We hypothesized that uric acid and lymphocyte count can be used in unison to improve the predictive power. Therefore, the present study was designed to evaluate the prognostic role of uric acid to lymphocyte count ratio (ULR) for adverse outcomes in elderly patients with RHD undergoing VRS.

\section{MATERIALS AND METHODS \\ Subjects}

The study was retrospectively conducted in consecutive patients with RHD undergoing VRS. A total of 1115 elderly patients (age $\geq 60$ years) were recruited in the Guangdong General Hospital from March 2009 to December 2015. All patients received at least 1 valve replacement. The exclusion criteria were as follows: (1) age less than 60 years; (2) history of malignant tumor; (3) previous diagnosis of gout; and (4) missed preoperative serum uric acid or lymphocyte count records. This study was approved by the Ethics Committee of our hospital with a waiver of informed consent because of the retrospective study design. Oral informed consent was obtained from patients or their relatives by telephone and recorded by trained nurses during the follow-up period.

\section{Examination and Data Collection}

Blood count and other variables, such as serum creatinine and electrolyte levels, were analyzed during hospital admission. Uric acid and other standard clinical parameters were routinely measured on the morning of the first day after admission in our center. Uric acid was automatically detected by the Beckman Coulter AU5800 (Beckman Coulter Inc, Brea Calif), and lymphocyte count was measured by the impedance method using an automated blood cell counter (XE-5000, Sysmex, Kobe, Japan). ULR was calculated as follows: serum uric acid (mg/dL)/lymphocyte count $\left(\times 10^{9} / \mathrm{L}\right)$. Transthoracic echocardiography was routinely performed before surgery, and left ventricular end-diastolic dimension, right ventricular (RV) diameter, left ventricular ejection fraction (LVEF), pulmonary systolic pressure (PSP), and other parameters were recorded.

Clinical information was collected from an electronic case report form by 1 researcher and randomly confirmed by another researcher. The European System for Cardiac Operative Risk Evaluation (euroSCORE) was calculated according to the criteria in this analysis. ${ }^{12}$

\section{Follow-up and End Points}

After discharge, surviving patients were followed up by telephone interview, examination of readmission clinical records, and outpatient clinic interviews. The primary end point was in-hospital death. One-year postoperative mortality and in-hospital major adverse clinical events (MACE), including dialysis, stroke, or death, were considered as the secondary end points The incidence of postoperative acute renal failure was also recorded, which was defined according to RIFLE criteria ${ }^{13}$ as an increase in serum creatinine to more than 3-fold from baseline, an increase in serum creatinine to more than $354 \mu \mathrm{mol} / \mathrm{L}$, or an acute increase of at least $44 \mu \mathrm{mol} / \mathrm{L}$.

\section{Statistical Analysis}

The SPSS program (SPSS Inc, Chicago, Ill) was used for all analyses. The results are presented as means ( \pm standard deviation) for continuous variables or percentages for categoric variables. The chi-square or Fisher exact test was used to compare categoric variables and variance or Wilcoxon rank-sum test to compare continuous variables. Cumulative rate of 1-year mortality was shown using Kaplan-Meier curves and compared using the log-rank test. Univariate and multivariable logistic regression analyses and Cox survival models were carried out to explore the relationship of variables with in-hospital and 1-year mortality, respectively. All significant variables in the univariate analysis were included in the multivariable model.

The bootstrap method with 1000 replications was used to perform an internal cross-validation of the ULR. The optimal cut-off was evaluated by receiver operator characteristic (ROC) curve and the average of area under the curve (AUC) was reported. The AUC was compared using the nonparametric approach of DeLong and colleagues. ${ }^{14}$ 


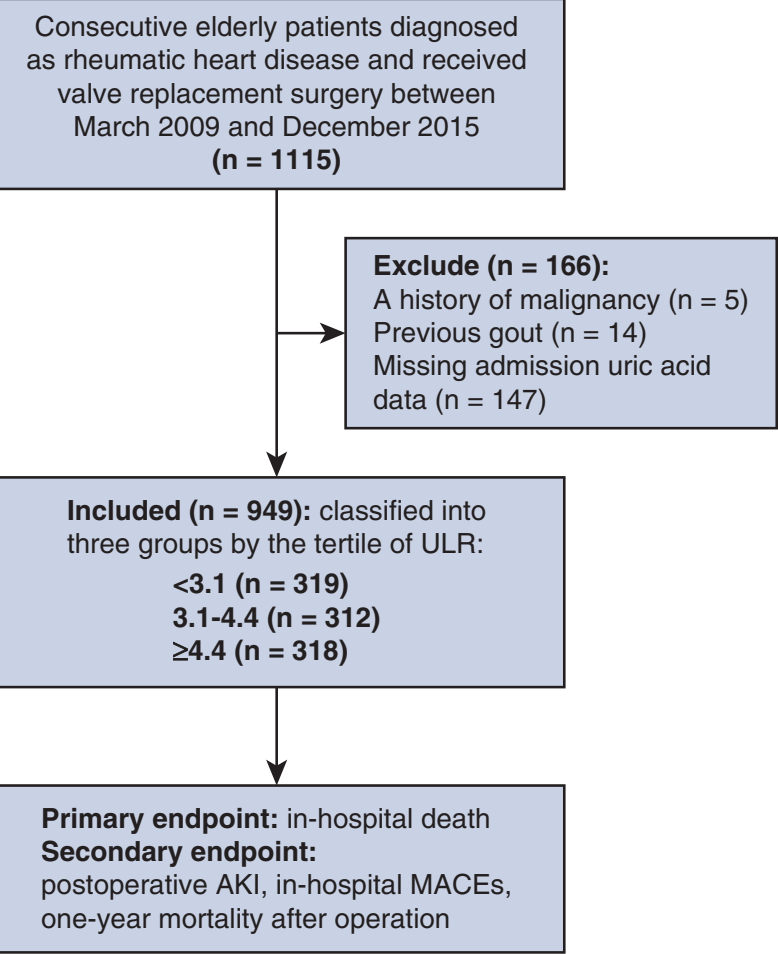

FIGURE 1. Patient disposition and selection of study cohort. ULR, Uric acid to lymphocyte count ratio; $A K I$, acute kidney injury; $M A C E$, major adverse clinical events.

\section{RESULTS}

\section{All Patients}

There were 1115 elderly patients with RHD who underwent VRS in our database, among whom 949 were finally included per our predefined exclusion criteria (Figure 1). The mean \pm standard deviation age of the whole cohort was $64 \pm 4$ years, and $64.7 \%$ of patients were female. During the hospital stay, 56 patients $(5.9 \%)$ died (cardiac death in 41$), 19$ patients $(2.0 \%)$ had a stroke, and 46 patients $(4.8 \%)$ received dialysis treatment.

\section{Development and Validation of Uric Acid to Lymphocyte Count Ratio}

An ROC curve analysis was conducted to determine the predictive value of uric acid, lymphocyte count, the combination by including both uric acid and lymphocyte count in the logistic model (the linear combination predictor), and ULR for in-hospital death. When compared with uric acid (AUC, 0.668; 95\% confidence interval [CI], 0.590-0.746; $P<.001$ ) (Figure 2) and lymphocyte count (AUC, 0.637; 95\% CI, 0.555-0.719; $P=.001$ ) (Figure 2), ULR (AUC, $0.703 ; 95 \%$ CI, 0.630-0.776; $P<.001$ ) (Figure 2) showed a higher predictive value for in-hospital mortality (compared with uric acid and lymphocyte count: $P=.334$ and .030 , respectively), although it was not significant when compared with uric acid. The ULR also had a similar

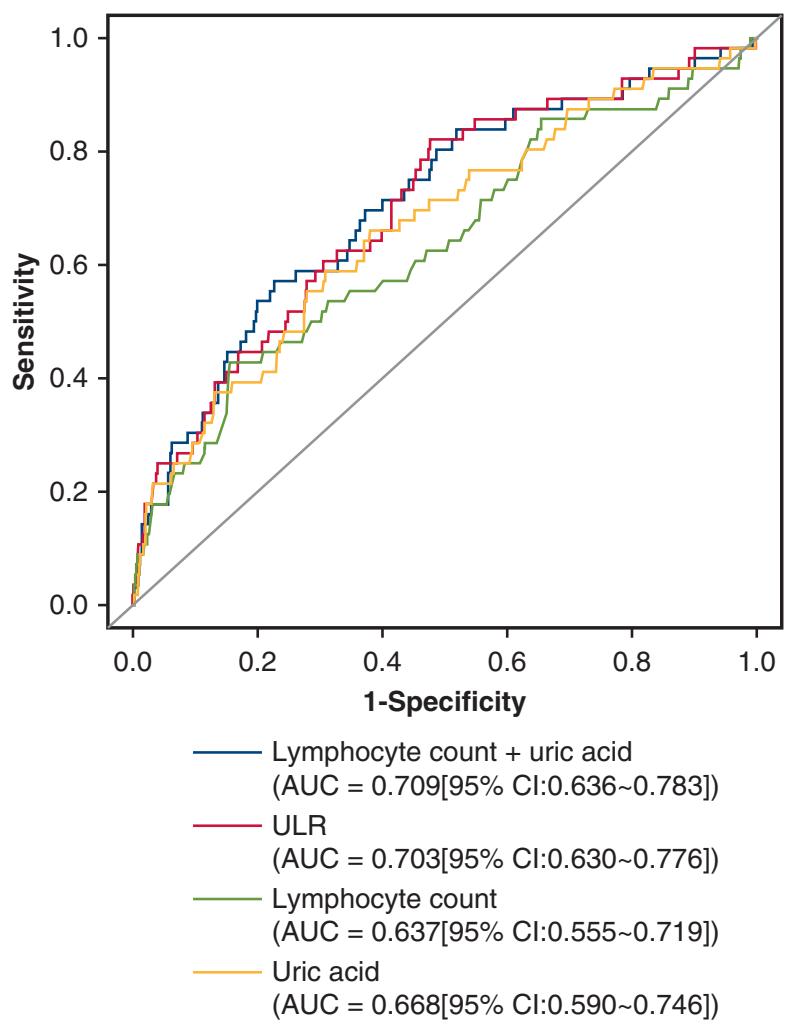

FIGURE 2. ROC curves of ULR, uric acid, lymphocyte count, and the prediction score developed by including the uric acid and lymphocyte count in the logistic model for in-hospital mortality. AUC, Area under the curve; $C I$, confidence interval; $U L R$, uric acid to lymphocyte count ratio.

AUC with the linear combination predictor (AUC, 0.709; 95\% CI, 0.636-0.783; $P<.001$ ) (Figure 2), but, for easier clinical use, we preferred the ULR. The bootstrapping internal validation for the ULR yielded an average AUC of 0.704 (95\% CI, 0.640-0.763) and was calibrated with a HosmerLemeshow chi-square statistic of $12.839(P=.118)$.

We also compared ULR with the EuroSCORE in 903 patients with available EuroSCORE. ULR showed an insignificantly higher predictive ability than the EuroSCORE (AUC, 0.701 vs $0.629, P=.141$ ) (Figure E1).

\section{Uric Acid to Lymphocyte Count Ratio and Clinical Characteristics}

Included patients were stratified into 3 groups by the tertile of ULR: less than $3.1(n=319), 3.1$ to $4.4(n=312)$, and 4.4 or greater $(n=318)$. Table 1 shows the baseline clinical characteristics of included patients. Patients in the first ULR tertile were significantly younger $(63.7 \pm 3.3$ years vs $64.2 \pm 3.5$ years vs $64.5 \pm 4.0$ years, $P=.028$ ). Patients with a higher ULR were more likely to be male. A positive trend with the proportion of New York Heart Association (NYHA) class III and IV, anemia, serum creatinine, uric acid, and C-reactive protein was 
TABLE 1. Baseline characteristics stratified by tertile of uric acid to lymphocyte count ratio

\begin{tabular}{|c|c|c|c|c|}
\hline Clinical variables & $\begin{array}{c}\text { First ULR tertile } \\
(<\mathbf{3 . 1}, \mathbf{n}=\mathbf{3 1 9})\end{array}$ & $\begin{array}{l}\text { Second ULR tertile } \\
(3.1-4.4, n=312)\end{array}$ & $\begin{array}{l}\text { Third ULR tertile } \\
\qquad(\geq 4.4, n=318)\end{array}$ & $P$ value \\
\hline Age (y) & $63.7 \pm 3.3$ & $64.2 \pm 3.5$ & $64.5 \pm 4.0$ & .028 \\
\hline Women, n (\%) & $241(75.5)$ & $195(62.5)$ & $178(56.0)$ & $<.001$ \\
\hline \multicolumn{5}{|l|}{ Concomitant disorders, $\mathrm{n}(\%)$} \\
\hline Diabetes mellitus & $21(6.6)$ & $31(9.9)$ & 34 (10.7) & .158 \\
\hline Hypertension & 54 (16.9) & $64(20.5)$ & $70(22.0)$ & .255 \\
\hline NYHA class III-IV, n (\%) & $118(37.0)$ & $130(41.7)$ & $160(50.3)$ & .003 \\
\hline Serum creatinine $(\mu \mathrm{mol} / \mathrm{L})$ & $74.3 \pm 18.4$ & $82.7 \pm 27.5$ & $94.5 \pm 32.1$ & $<.001$ \\
\hline WBC count $\left(* 10^{9} / \mathrm{L}\right)$ & $7.2 \pm 1.9$ & $6.6 \pm 1.7$ & $6.3 \pm 1.7$ & $<.001$ \\
\hline Neutrophil count $\left(* 10^{9} / \mathrm{L}\right)$ & $3.9 \pm 1.5$ & $3.9 \pm 1.4$ & $4.0 \pm 1.5$ & .256 \\
\hline Lymphocyte count $\left(* 10^{9} / \mathrm{L}\right)$ & $2.5 \pm 0.6$ & $2.0 \pm 0.4$ & $1.4 \pm 0.4$ & $<.001$ \\
\hline Hemoglobin $(\mathrm{g} / \mathrm{L})$ & $134.5 \pm 14.0$ & $132.1 \pm 17.1$ & $130.1 \pm 17.5$ & .003 \\
\hline Anemia, n (\%) & $19(6.0)$ & 33 (10.6) & $50(15.7)$ & $<.001$ \\
\hline Uric acid $(\mathrm{mg} / \mathrm{dL})$ & $5.9 \pm 1.4$ & $7.2 \pm 1.5$ & $8.7 \pm 2.1$ & $<.001$ \\
\hline CRP (mg/L) & $2.5(1.7-4.3)$ & $2.8(1.8-5.4)$ & $3.7(2.1-6.3)$ & $<.001$ \\
\hline LVEDD (mm) & $48.4 \pm 7.6$ & $48.8 \pm 8.3$ & $50.4 \pm 9.7$ & .014 \\
\hline RV diameter (mm) & $50.0 \pm 5.8$ & $51.1 \pm 6.2$ & $54.0 \pm 7.9$ & $<.001$ \\
\hline LVEF (\%) & $62.8 \pm 8.1$ & $62.6 \pm 8.7$ & $60.0 \pm 10.3$ & $<.001$ \\
\hline PSP (mm Hg) & $39.4 \pm 14.0$ & $44.9 \pm 19.5$ & $50.6 \pm 20.3$ & $<.001$ \\
\hline \multicolumn{5}{|l|}{ Valve surgery } \\
\hline AVR & $139(43.6)$ & $144(46.2)$ & $163(51.3)$ & .142 \\
\hline MVR & $295(92.5)$ & $290(92.9)$ & $303(95.3)$ & .303 \\
\hline TVI & $223(69.9)$ & $228(73.1)$ & $262(82.4)$ & .001 \\
\hline $\mathrm{AVR}+\mathrm{MVR}$ & $116(36.4)$ & $124(39.7)$ & $153(48.1)$ & .008 \\
\hline $\mathrm{AVR}+\mathrm{MVR}+\mathrm{TVI}$ & 89 (27.9) & $97(31.1)$ & $124(39.0)$ & .009 \\
\hline CABG surgery & $22(6.9)$ & $29(9.3)$ & $19(6.0)$ & .259 \\
\hline EuroSCORE & $4.4 \pm 1.0$ & $4.5 \pm 1.2$ & $5.0 \pm 1.5$ & $<.001$ \\
\hline Postoperative renal failure & $130(40.8)$ & $148(47.6)$ & $170(53.6)$ & .005 \\
\hline
\end{tabular}

$U L R$, Uric acid to lymphocyte count ratio; $N Y H A$, New York Heart Association; $W B C$, white blood cell; $C R P, \mathrm{C}$-reactive protein; $L V E D D$, left ventricular end-diastolic dimension; $R V$, right ventricular; $L V E F$, left ventricular ejection fraction; $P S P$, pulmonary systolic pressure; $A V R$, aortic valve replacement; $M V R$, mitral valve replacement; TVI, tricuspid valve intervention; $C A B G$, coronary artery bypass grafting; EuroSCORE, European System for Cardiac Operative Risk Evaluation.

observed with increasing ULR. However, a negative trend with white blood cell count, lymphocyte count, hemoglobin, and LVEF was observed with increasing ULR. Higher left ventricular end-diastolic dimension, RV diameter, and PSP were found in patients in the upper tertile. In addition, there was a trend among tertiles toward a higher incidence of tricuspid valve intervention as ULR increased. The data for assessing postoperative renal failure were available in 947 patients. The incidence of postoperative renal failure was $47.3 \%$ (448/947), which is significantly higher in patients in the upper tertile $(40.8 \%$ vs $47.6 \%$ vs $53.6 \%$, $P=.005$ ) (Table 1).

\section{Uric Acid to Lymphocyte Count Ratio and In-Hospital Clinical Outcomes}

The risk factors for in-hospital death according to univariate and multivariate logistic regression analyses are presented in Table 2. The univariate analysis revealed that male patients and patients with a high ULR, older age, a high proportion of NYHA class III and IV, estimated glomerular filtration rate (eGFR) less than $60 \mathrm{~mL} / \mathrm{min} /$ $1.73 \mathrm{~m}^{2}$, anemia, a high RV diameter, a high PSP, and low LVEF were at higher risk for in-hospital death. In the multivariate analysis, ULR was the only significant independent indicator for in-hospital death (odds ratio [OR], 1.17; 95\% CI, 1.07-1.29; $P=.001)$.

\section{Uric Acid to Lymphocyte Count Ratio and One-Year Postoperative Mortality}

Patients were followed up for 1 year after VRS. In this time, 69 patients died and 88 patients were lost to followup. In univariate Cox proportional hazards analysis, ULR was significantly associated with 1-year postoperative mortality (unadjusted hazard ratio $[\mathrm{HR}], 1.19 ; P<.001$, 
TABLE 2. Results of logistic regression analysis for in-hospital mortality

\begin{tabular}{|c|c|c|c|c|}
\hline \multirow[b]{2}{*}{ Clinical variables } & \multicolumn{2}{|c|}{ Univariate } & \multicolumn{2}{|c|}{ Multivariate } \\
\hline & $\overline{\text { OR }}$ & $P$ & OR $(95 \%$ CI $)$ & $P$ \\
\hline ULR & 1.24 & $<.001$ & $1.17(1.07-1.29)$ & .001 \\
\hline Age (y) & 1.08 & .019 & $1.06(0.98-1.13)$ & .144 \\
\hline Women & 0.57 & .039 & $0.73(0.40-1.32)$ & .296 \\
\hline Diabetes mellitus & 1.22 & .658 & & \\
\hline Hypertension & 0.76 & .470 & & \\
\hline NYHA class III-IV & 2.32 & .003 & $1.62(0.90-2.93)$ & .110 \\
\hline $\mathrm{eGFR}<60 \mathrm{~mL} / \mathrm{min} / 1.73 \mathrm{~m}^{2}$ & 2.15 & .010 & $1.14(0.57-2.27)$ & .707 \\
\hline WBC count & 0.93 & .352 & & \\
\hline Neutrophil count & 1.03 & .732 & & \\
\hline Anemia & 2.73 & .003 & $1.60(0.77-3.32)$ & .206 \\
\hline $\log _{10} \mathrm{CRP}$ & 1.75 & .093 & & \\
\hline LVEDD & 1.02 & .227 & & \\
\hline RV diameter & 1.06 & $<.001$ & $1.01(0.97-1.05)$ & .710 \\
\hline LVEF & 0.97 & .016 & $0.98(0.95-1.01)$ & .129 \\
\hline PSP & 1.02 & $<.001$ & $1.02(1.00-1.03)$ & .046 \\
\hline AVR & 1.23 & .460 & & \\
\hline MVR & 0.89 & .822 & & \\
\hline TVI & 1.56 & .214 & & \\
\hline $\mathrm{AVR}+\mathrm{MVR}$ & 1.24 & .433 & & \\
\hline $\mathrm{AVR}+\mathrm{MVR}+\mathrm{TVI}$ & 1.06 & .835 & & \\
\hline CABG surgery & 1.55 & .328 & & \\
\hline $\begin{array}{l}\text { OR, Odds ratio; } C I \text {, confidence in } \\
N Y H A \text {, New York Heart Associa } \\
W B C \text {, white blood cell; } C R P \text {, } \\
\text { diastolic dimension; } R V \text {, right ve } \\
P S P \text {, pulmonary systolic pressure; } \\
\text { replacement; } T V I \text {, tricuspid val } \\
\text { grafting. }\end{array}$ & $\begin{array}{l}\text { erval; } L \\
\text { on; } e G \\
\text { reactive } \\
\text { ricular; } \\
I V R \text {, aor } \\
\text { interv }\end{array}$ & $\begin{array}{l}R \text {, uric a } \\
R \text {, estima } \\
\text { protein; } \\
L V E F, \text { lef } \\
\text { ic valve } \mathrm{r} \\
\text { ntion; } C\end{array}$ & $\begin{array}{l}\mathrm{d} \text { to lymphocyte cou } \\
\text { ed glomerular filtrati } \\
V E D D \text {, left ventricul } \\
\text { ventricular ejection } \\
\text { lacement; } M V R \text {, mitr } \\
B G \text {, coronary artery }\end{array}$ & $\begin{array}{l}\text { t ratio; } \\
\text { on rate; } \\
\text { ar end- } \\
\text { raction; } \\
\text { al valve } \\
\text { bypass }\end{array}$ \\
\hline
\end{tabular}

Table 3). Other significant parameters included age, sex, NYHA class III and IV, eGFR less than $60 \mathrm{~mL} / \mathrm{min} /$ $1.73 \mathrm{~m}^{2}$, anemia, $\log _{10} \mathrm{C}$-reactive protein, $\mathrm{RV}$ diameter, LVEF, and PSP. After adjusting for these potential risk factors, ULR remained as the only independent predictor for 1year postoperative death (adjusted HR, 1.13; 95\% CI, 1.03$1.25 ; P=.010$ ) (Table 3 ).

\section{Clinical Application of Uric Acid to Lymphocyte Count Ratio}

We arbitrarily categorized the risk scores into 3 groups by the tertile of ULR to enhance its clinical use: low risk, moderate risk, and high risk. The in-hospital mortality ( $2.2 \%$ vs $4.8 \%$ vs $10.7 \%, P<.001)$ (Figure $3, A)$, MACE $(3.4 \%$ vs $8.0 \%$ vs $13.2 \%, P<.001$ ) (Figure $3, A$ ), and 1 -year mortality $(2.2 \%$ vs $6.4 \%$ vs $13.2 \%, P<.001$ ) (Figure $3, A$ ) increased from the lowest to the highest ULR tertile. Compared with the first ULR tertile, the third ULR tertile
TABLE 3. Results of Cox proportional hazards model for postoperative 1-year mortality

\begin{tabular}{|c|c|c|c|c|}
\hline \multirow[b]{2}{*}{ Clinical variables } & \multicolumn{2}{|c|}{ Univariate } & \multicolumn{2}{|c|}{ Multivariate } \\
\hline & $\overline{\text { HR }}$ & $P$ & HR $(95 \%$ CI $)$ & $P$ \\
\hline ULR & 1.19 & $<.001$ & $1.13(1.03-1.25)$ & .010 \\
\hline Age $(y)$ & 1.07 & .030 & $1.02(0.96-1.10)$ & .505 \\
\hline Women & 0.53 & .009 & $0.71(0.41-1.21)$ & .209 \\
\hline Diabetes mellitus & 1.11 & .789 & & \\
\hline Hypertension & 0.68 & .254 & & \\
\hline NYHA class III-IV & 1.92 & .007 & $1.17(0.69-1.97)$ & .564 \\
\hline $\mathrm{eGFR}<60 \mathrm{~mL} / \mathrm{min} / 1.73 \mathrm{~m}^{2}$ & 2.12 & .004 & $1.07(0.58-1.98)$ & .839 \\
\hline WBC count & 0.97 & 683 & & \\
\hline Neutrophil count & 1.09 & .258 & & \\
\hline Anemia & 2.61 & .001 & $1.91(1.02-3.58)$ & .044 \\
\hline $\log _{10} \mathrm{CRP}$ & 1.98 & .014 & $0.99(0.55-1.78)$ & .962 \\
\hline LVEDD & 1.02 & .176 & & \\
\hline RV diameter & 1.07 & $<.001$ & $1.02(0.98-1.06)$ & .365 \\
\hline LVEF & 0.96 & .001 & $0.99(0.96-1.02)$ & .389 \\
\hline PSP & 1.02 & $<.001$ & $1.01(1.00-1.03)$ & .032 \\
\hline AVR & 1.33 & .243 & & \\
\hline MVR & 0.73 & .457 & & \\
\hline TVI & 1.29 & .389 & & \\
\hline $\mathrm{AVR}+\mathrm{MVR}$ & 1.25 & .365 & & \\
\hline AVR + MVR + TVI & 1.03 & .909 & & \\
\hline CABG surgery & 1.45 & .351 & & \\
\hline
\end{tabular}

$H R$, Hazard ratio; $C I$, confidence interval; $U L R$, uric acid to lymphocyte count ratio; NYHA, New York Heart Association; $e G F R$, estimated glomerular filtration rate; $W B C$, white blood cell; $C R P$, C-reactive protein; $L V E D D$, left ventricular enddiastolic dimension; $R V$, right ventricular; $L V E F$, left ventricular ejection fraction; $P S P$, pulmonary systolic pressure; $A V R$, aortic valve replacement; $M V R$, mitral valve replacement; TVI, tricuspid valve intervention; $C A B G$, coronary artery bypass grafting.

was an independent predictor for in-hospital mortality (adjusted OR, 2.71; 95\% CI, 1.10-6.66; $P=.030$ ) (Table E1) and 1-year mortality (adjusted HR, 2.98; 95\% CI, 1.25-7.09; $P=.014$ ) (Table E1). Compared with lowrisk patients, the cumulative 1-year postoperative mortality risk was significantly higher in moderate-risk and high-risk patients $(\log -$ rank $=30.21, P<.001)($ Figure $4, A)$.

In addition, we also divided the patients into 2 risk levels using a cutoff of 3.7 (sensitivity $82.1 \%$; specificity $52.4 \%$ ) selected according to the ROC curve. The in-hospital mortality $(2.3 \%$ vs $9.6 \%, P<.001)$ (Figure $3, B)$, MACE $(4.2 \%$ vs $12.4 \%, P<.001$ ) (Figure $3, B$ ), and 1-year mortality $(2.7 \%$ vs $11.9 \%, P<.001)$ (Figure $3, B)$ were significantly higher in patients with ULR greater than 3.7. ULR greater than 3.7 was independently associated with in-hospital (adjusted OR, 2.69; 95\% CI, 1.30-5.56; $P=.007$, Table E1) and 1-year mortality (adjusted HR, 2.52; 95\% CI, $1.30-4.87 ; P=.006$ ) (Table E1). Patients with ULR greater 


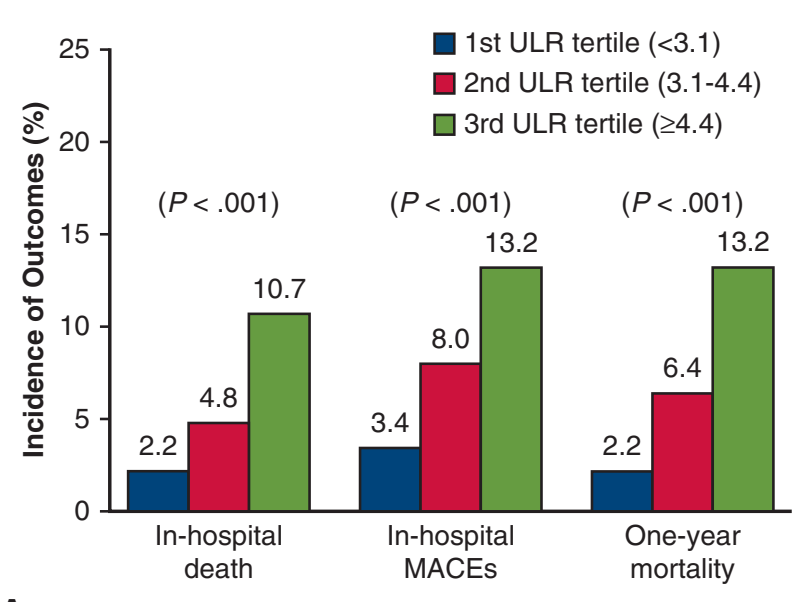

A

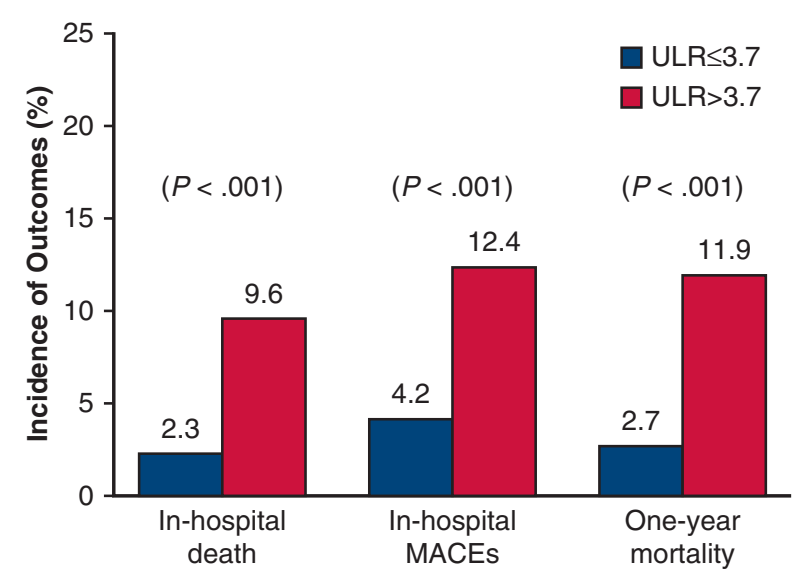

B

FIGURE 3. Prevalence of adverse events. MACE, Major adverse clinical events; $U L R$, uric acid to lymphocyte count ratio.

than 3.7 had worse 1-year prognosis than those without $(\log$-rank $=31.15, P<.001)($ Figure $4, B)$.

\section{DISCUSSION}

To our best knowledge, this study was the first to use ULR as a preoperative risk-stratified method. We found that ULR produced relatively higher prognostic value than uric acid or lymphocyte count, and was independently associated with in-hospital and 1-year mortality in elderly patients with RHD undergoing VRS. In addition, the predictive ability of ULR was similar to the EuroSCORE. The use of ULR could provide prognostic information for early and fast preoperative risk assessment in elderly patients with RHD undergoing VRS.

In this study, 949 elderly patients with RHD were retrospectively analyzed, and all underwent at least 1 VRS because of severe valve damage with symptoms of cardiac dysfunction. Oxidative stress, which triggered by activating the sympathetic nervous and the renin-angiotensinaldosterone system, played a critical role in the pathophysiology of heart failure. ${ }^{15,16}$ In this condition, reactive oxygen species including xanthine oxidase were excessively produced. Xanthine oxidase is an important catalyzing enzyme, which induced the formation of uric acid from xanthine and hypoxanthine. ${ }^{17}$ In addition, uric acid would be released in hypoxic conditions. ${ }^{18}$ Hyperuricemia might be due to hypoxia and impaired oxidative metabolism in heart failure. A decrease in renal perfusion in heart failure was another mechanism for increasing serum uric acid levels. Uric acid is primarily excreted through the kidney. More than $50 \%$ of patients with acute and chronic heart failure have renal dysfunction. ${ }^{19}$ In our study, $74.1 \%$ of included patients had existing renal insufficiency $\left(\right.$ GFR $<90 \mathrm{~mL} / \mathrm{min} / 1.73 \mathrm{~m}^{2}$ ). Decline in cardiac output, activation of neurohormonal systems, or inflammation in heart failure contributed to impaired renal function, which subsequently diminished the excretion of uric acid. ${ }^{20}$ Hyperuricemia was a marker of excessive oxidative stress and severity of renal dysfunction, which was established as a predictor for adverse prognosis in several conditions. ${ }^{21-23}$ Inflammation also involved the pathogenesis and progression of heart failure. ${ }^{24,25}$ Uric acid increased in keeping with the level of inflammatory markers in chronic heart failure, which represented the severity of cardiac dysfunction.

Decreased lymphocyte count was another phenomenon in patients with heart failure because of increased neurohormonal activation. Increased cortisol and catecholamines were postulated to be the underlying mechanism causing lymphopenia by triggering apoptosis and downregulating the proliferation and differentiation of lymphocytes. ${ }^{26,27}$ In addition, RHD is an autoimmune disease and inflammatory response still persists in its chronic phase..$^{28}$ Further, inflammation and immune response were important elements in the pathogenesis and progression of heart failure. ${ }^{29}$ The over-release of proinflammatory cytokines may be directly related to lowered lymphocyte counts. ${ }^{30}$ Another possible mechanism was intestinal lymphangiectasia, which is caused by systemic venous and lymphatic hypertension. This lymphangiectasia triggered loss of lymphocyte-rich lymph into the gastrointestinal tract, resulting in lymphopenia.

In this study, we only focused on elderly patients, whose organ function had declined with age. Few preoperative risk-stratified methods were emphasized in this special population. Epidemiologic data revealed that the prevalence of chronic kidney disease in the elderly is estimated to be $40 \%{ }^{31}$ This renal dysfunction contributed to hyperuricemia. On the other hand, reduced lymphocyte count and its function were common in elderly patients and mostly depend on nutritional status. ${ }^{32,33}$ Hyperuricemia and reduced lymphocyte count were established predictors for poor outcomes in heart failure. ${ }^{8,34}$ These mechanisms encouraged us to combine these 2 parameters into a new 


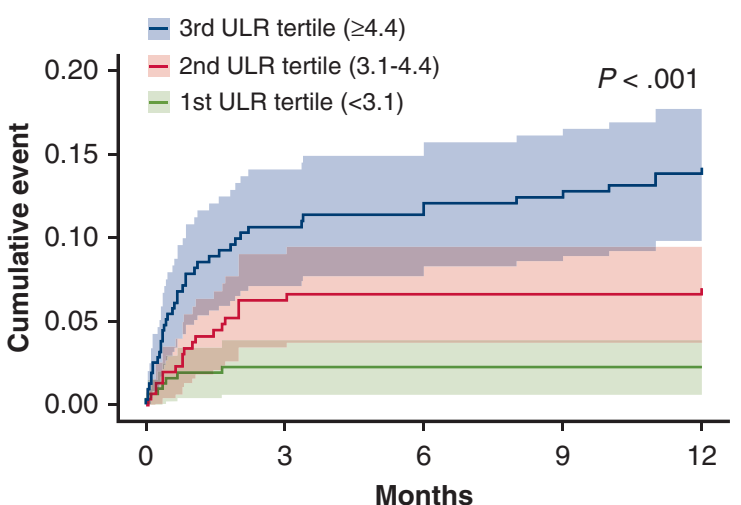

Number at risk 1st ULR tertile 2nd ULR tertile 312 3rd ULR tertile 318

A

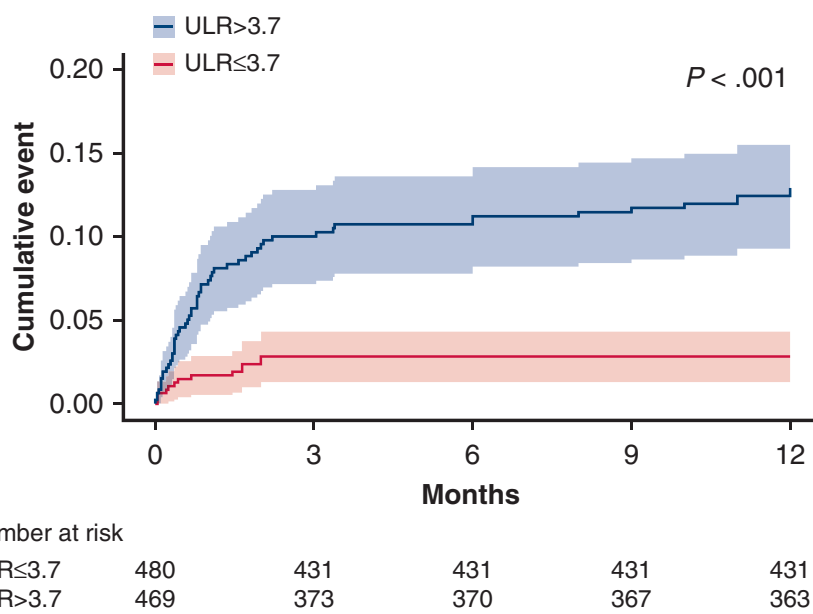

B

FIGURE 4. Cumulative incidence curve for 1-year postoperative death. A, Classified by the tertiles of ULR. B, Classified by the receiver operating characteristic curve selected cut-off of ULR. ULR, Uric acid to lymphocyte count ratio.

variable as a preoperative risk-evaluated method in elderly patients with RHD undergoing VRS. The ULR appeared to be a more powerful predictor than either parameter alone, which could identify more patients at high risk of postoperative death.

\section{Study Limitations}

Our study has a few limitations. First, although a multivariate analysis was performed to adjust the potential confounding factors, some residual factors might have had an impact on the end points because of the retrospective study design. Second, we did not investigate the reasons for preoperative hyperuricemia and reduced lymphocyte count. However, this study aimed to identify a novel marker and its prognostic value in elderly patients with RHD undergoing VRS. Third, ULR had a higher ability than uric acid for predicting in-hospital death, but it did not reach statistical significance probably because of the small sample size. In

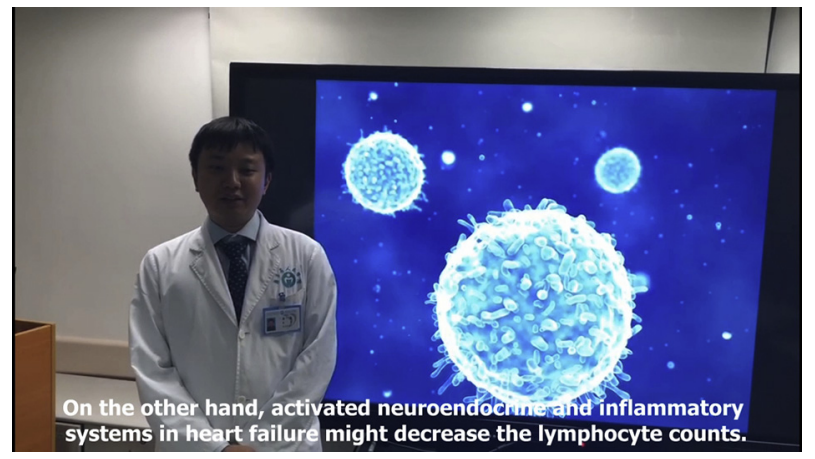

VIDEO 1. The importance and relevance of the article are explained. Video available at: https://www.jtcvs.org/article/S0022-5223(18)32817-4/fulltext. the later study, we will continue to increase the sample size to determine whether ULR is more powerful than uric acid. Finally, the effect of ULR was explored only in elderly patients. Whether it can be extrapolated to other age groups in the population remains to be seen.

\section{CONCLUSIONS}

To our best knowledge, this study was the first to combine 2 distinct phenomena-hyperuricemia and reduced lymphocyte count-to predict adverse prognosis among elderly patients with RHD undergoing VRS. This novel index, ULR, produced relatively higher predictive power and was independently associated with in-hospital and 1-year mortality, which could be considered as an early and fast preoperative risk assessment tool. Emphasis should be placed on those elderly patients with RHD and preoperative high ULR (Video 1).

\section{Conflict of Interest Statement}

Authors have nothing to disclose with regard to commercial support.

\section{References}

1. Marijon E, Mirabel M, Celermajer DS, Jouven X. Rheumatic heart disease. Lancet. 2012;379:953-64.

2. Nishimura RA, Otto CM, Bonow RO, Carabello BA, Erwin JR, Fleisher LA, et al. 2017 AHA/ACC focused update of the 2014 AHA/ACC guideline for the management of patients with valvular heart disease: a report of the American College of Cardiology/American Heart Association task force on clinical practice guidelines. J Am Coll Cardiol. 2017;70:252-89.

3. Rusingiza EK, El-Khatib Z, Hedt-Gauthier B, Ngoga G, Dusabeyezu S, Tapela N, et al. Outcomes for patients with rheumatic heart disease after cardiac surgery followed at rural district hospitals in Rwanda. Heart. 2018;104:1707-13.

4. Wei XB, Jiang L, Liu YH, Feng D, He PC, Chen JY, et al. Thrombocytopenia as a preoperative risk assessment tool in patients with rheumatic heart disease undergoing valve replacement surgery. J Am Heart Assoc. 2017;6. 
5. Palazzuoli A, Ruocco G, De Vivo O, Nuti R, McCullough PA. Prevalence of hyperuricemia in patients with acute heart failure with either reduced or preserved ejection fraction. Am J Cardiol. 2017;120:1146-50.

6. Kaufman M, Guglin M. Uric acid in heart failure: a biomarker or therapeutic target? Heart Fail Rev. 2013;18:177-86

7. Leyva F, Anker SD, Godsland IF, Teixeira M, Hellewell PG, Kox WJ, et al. Uric acid in chronic heart failure: a marker of chronic inflammation. Eur Heart J. 1998;19:1814-22.

8. Mantovani A, Targher G, Temporelli PL, Lucci D, Gonzini L, Nicolosi GL, et al. Prognostic impact of elevated serum uric acid levels on long-term outcomes in patients with chronic heart failure: a post-hoc analysis of the GISSI-HF (Gruppo Italiano per lo Studio della Sopravvivenza nella Insufficienza Cardiaca-Heart Failure) trial. Metabolism. 2018;83:205-15.

9. Huang WM, Hsu PF, Cheng HM, Lu DY, Cheng YL, Guo CY, et al. Determinants and prognostic impact of hyperuricemia in hospitalization for acute heart failure. Circ J. 2016;80:404-10.

10. Shimizu T, Yoshihisa A, Kanno Y, Takiguchi M, Sato A, Miura S, et al. Relationship of hyperuricemia with mortality in heart failure patients with preserved ejection fraction. Am J Physiol Heart Circ Physiol. 2015;309:H1 123-9.

11. Vaduganathan M, Ambrosy AP, Greene SJ, Mentz RJ, Subacius HP, Maggioni AP, et al. Predictive value of low relative lymphocyte count in patients hospitalized for heart failure with reduced ejection fraction: insights from the EVEREST trial. Circ Heart Fail. 2012;5:750-8.

12. Roques F, Nashef SA, Michel P, Gauducheau E, de Vincentiis C, Baudet E, et al. Risk factors and outcome in European cardiac surgery: analysis of the EuroSCORE multinational database of 19030 patients. Eur J Cardiothorac Surg. 1999; 15:816-22; discussion 822-3

13. Bellomo R, Ronco C, Kellum JA, Mehta RL, Palevsky P, Acute Dialysis Quality Initiative workgroup. Acute renal failure - definition, outcome measures, animal models, fluid therapy and information technology needs: the second international consensus conference of the acute dialysis quality initiative (ADQI) group. Crit Care. 2004;8:R204-12.

14. DeLong ER, DeLong DM, Clarke-Pearson DL. Comparing the areas under two or more correlated receiver operating characteristic curves: a nonparametric approach. Biometrics. 1988;44:837-45.

15. Munzel T, Gori T, Keaney JJ, Maack C, Daiber A. Pathophysiological role of oxidative stress in systolic and diastolic heart failure and its therapeutic implications. Eur Heart J. 2015;36:2555-64.

16. Wojciechowska C, Romuk E, Tomasik A, Skrzep-Poloczek B, NowalanyKozielska E, Birkner E, et al. Oxidative stress markers and C-reactive protein are related to severity of heart failure in patients with dilated cardiomyopathy. Mediators Inflamm. 2014;2014:147040.

17. Bergamini C, Cicoira M, Rossi A, Vassanelli C. Oxidative stress and hyperuricaemia: pathophysiology, clinical relevance, and therapeutic implications in chronic heart failure. Eur J Heart Fail. 2009;11:444-52.

18. Baillie JK, Bates MG, Thompson AA, Waring WS, Partridge RW, Schnopp MF, et al. Endogenous urate production augments plasma antioxidant capacity in healthy lowland subjects exposed to high altitude. Chest. 2007;131:1473-8.
19. Damman K, Testani JM. The kidney in heart failure: an update. Eur Heart $J$ 2015;36:1437-44.

20. Braam B, Joles JA, Danishwar AH, Gaillard CA. Cardiorenal syndrome-curren understanding and future perspectives. Nat Rev Nephrol. 2014;10:48-55.

21. Zuo T, Jiang L, Mao S, Liu X, Yin X, Guo L. Hyperuricemia and contrastinduced acute kidney injury: a systematic review and meta-analysis. Int J Cardiol. 2016;224:286-94.

22. Li M, Hou W, Zhang X, Hu L, Tang Z. Hyperuricemia and risk of stroke: a systematic review and meta-analysis of prospective studies. Atherosclerosis. 2014 232:265-70.

23. Braga F, Pasqualetti S, Ferraro S, Panteghini M. Hyperuricemia as risk factor for coronary heart disease incidence and mortality in the general population: a systematic review and meta-analysis. Clin Chem Lab Med. 2016;54:7-15.

24. Heidenreich P. Inflammation and heart failure: therapeutic or diagnostic opportunity? J Am Coll Cardiol. 2017;69:1286-7.

25. Dick SA, Epelman S. Chronic heart failure and inflammation: what do we really know? Circ Res. 2016;119:159-76.

26. Nunez J, Nunez E, Minana G, Sanchis J, Bodi V, Rumiz E, et al. Effectiveness of the relative lymphocyte count to predict one-year mortality in patients with acute heart failure. Am J Cardiol. 2011;107:1034-9.

27. Rudiger A, Burckhardt OA, Harpes P, Muller SA, Follath F. The relative lymphocyte count on hospital admission is a risk factor for long-term mortality in patients with acute heart failure. Am J Emerg Med. 2006; 24:451-4.

28. Golbasi Z, Ucar O, Keles T, Sahin A, Cagli K, Camsari A, et al. Increased levels of high sensitive C-reactive protein in patients with chronic rheumatic valve disease: evidence of ongoing inflammation. Eur J Heart Fail. 2002;4: 593-5.

29. Zhang Y, Bauersachs J, Langer HF. Immune mechanisms in heart failure. Eur J Heart Fail. 2017;19:1379-89.

30. von Haehling S, Schefold JC, Jankowska E, Doehner W, Springer J, Strohschein K, et al. Leukocyte redistribution: effects of beta blockers in patients with chronic heart failure. PLoS One. 2009;4:e6411.

31. Coresh J, Selvin E, Stevens LA, Manzi J, Kusek JW, Eggers P, et al. Prevalence of chronic kidney disease in the United States. JAMA. 2007;298: 2038-47.

32. Lesourd B. Nutritional factors and immunological ageing. Proc Nutr Soc. 2006 65:319-25.

33. Ahluwalia N. Aging, nutrition and immune function. J Nutr Health Aging. 2004 8:2-6.

34. Marcula M, de Souza BM, Madaloso BA, Nunes RA, Cuoco MA, de Paula RS, et al. Lymphocyte count and prognosis in patients with heart failure. Int J Cardiol. 2015;188:60-2.

Key Words: lymphocyte, outcomes, rheumatic heart disease, uric acid, valve replacement surgery 


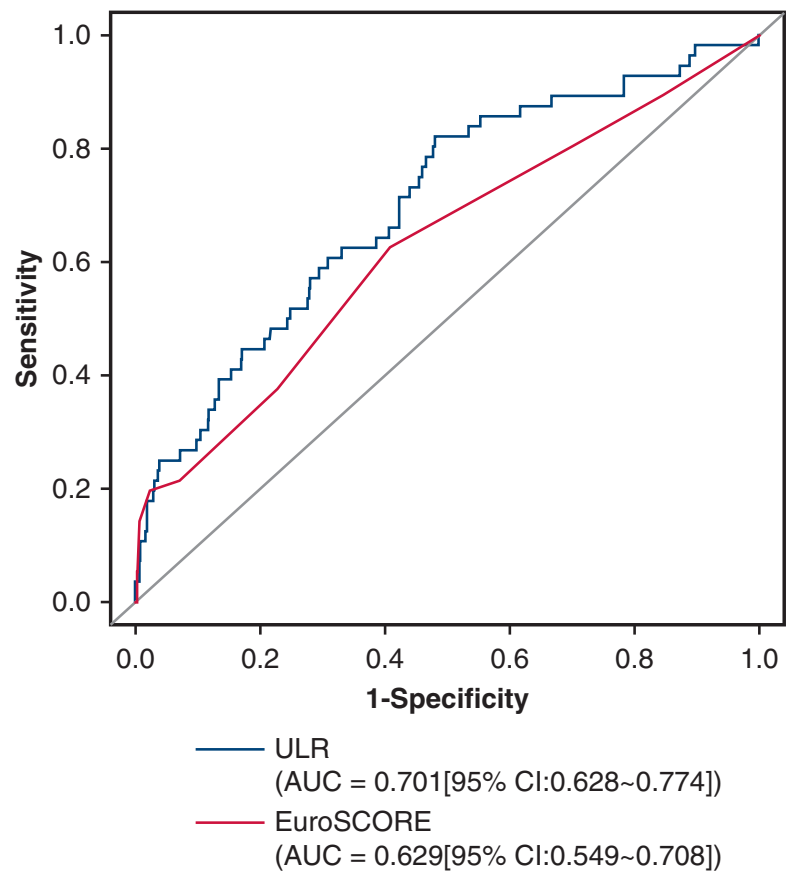

FIGURE E1. ROC curves of ULR and euroSCORE for in-hospital mortality (data for euroSCORE missed for 46 patients). $U L R$, Uric acid to lymphocyte count ratio; $A U C$, area under the curve; $C I$, confidence interval; EuroSCORE, European System for Cardiac Operative Risk Evaluation.

TABLE E1. Multivariable analysis for in-hospital and 1-year death

\begin{tabular}{|c|c|c|c|c|c|c|}
\hline \multirow[b]{2}{*}{ Variable } & \multicolumn{6}{|c|}{ ULR } \\
\hline & Second vs first tertile & $P$ & Third vs first tertile & $P$ & $>3.7$ vs $\leq 3.7$ & $P$ \\
\hline \multicolumn{7}{|l|}{ In-hospital death } \\
\hline Unadjusted OR (95\% CI) & $2.25(0.91-5.60)$ & .081 & $5.34(2.33-12.23)$ & $<.001$ & $4.53(2.31-8.86)$ & $<.001$ \\
\hline Adjusted OR $(95 \% \text { CI })^{*}$ & $1.70(0.67-4.31)$ & .264 & $2.71(1.10-6.66)$ & .030 & $2.69(1.30-5.56)$ & .007 \\
\hline \multicolumn{7}{|l|}{$1-y$ death } \\
\hline Unadjusted HR (95\% CI) & $3.08(1.30-7.29)$ & .010 & $6.56(2.95-14.59)$ & $<.001$ & $4.74(2.59-8.67)$ & $<.001$ \\
\hline Adjusted HR (95\% CI) $\dagger$ & $2.30(0.95-5.54)$ & .064 & $2.98(1.25-7.09)$ & .014 & $2.52(1.30-4.87)$ & .006 \\
\hline
\end{tabular}

$U L R$, Uric acid to lymphocyte count ratio; $O R$, odds ratio; $C I$, confidence interval; $H R$, hazard ratio. *Adjusted for age, sex, NYHA class III-IV, eGFR less than $60 \mathrm{~mL} / \mathrm{min} /$ $1.73 \mathrm{~m}^{2}$, anemia, RV diameter, LVEF, and PSP. †Adjusted for age, sex, NYHA class III and IV, eGFR less than $60 \mathrm{~mL} / \mathrm{min} / 1.73 \mathrm{~m}{ }^{2}$, anemia, $\log _{10}$ CRP, RV diameter, LVEF, and PSP. 